\title{
Booster Foods for Your Immune System: Healthy Notes to Consumers. A Short Review
}

\author{
Majid Mohammed Mahmood ${ }^{1 *}$ and Zenab Ghanim Younus Al-Ameen ${ }^{2}$ \\ ${ }^{1}$ Unit of Zoonotic Diseases, College of Veterinary Medicine, University of Baghdad, \\ Iraq \\ ${ }^{2}$ BPharm, College of Pharmacy, University of Baghdad, Iraq \\ *Corresponding Author: Majid Mohammed Mahmood, Unit of Zoonotic Diseases, \\ College of Veterinary Medicine, University of Baghdad, Iraq.
}

Received: April 15, 2021

Published: July 29, 2021

(C) All rights are reserved by Majid

Mohammed Mahmood and Zenab Ghanim Younus Al-Ameen.

\begin{abstract}
This review article aims at raising and enhancing immunity to confront SARS-CoV-2 virus (the causative agent of COVID-19 disease) using dietary approaches. Because there is no effective treatment although some medicines approved by FDA, or a successful protective vaccine, the choice to combat this disease and one of the solutions depends on preventing and elevating level of immunity. Therefore, this review reports available scientific evidence that healthy eating can help enhancing the immune system and prevent diseases. Targeting consumers globally to notice much important types of healthy food (mentioned in this review) may help to build up a healthy immune body resistant against viruses and other bugs.
\end{abstract}

Keywords: Immunity; Food; COVID-19; Boosting Immune System

\section{Introduction}

The indications, attributes and treatment options of the COVID-19 are getting increasingly comprehended as the worldwide pandemic progresses; but the importance of adapted nourishment in the protection process is often under assessed. Nutrient inadequacy such as micronutrients seem to correlate with decreased immune function and the contrast would be best in assisting with battling against infection [1].

General wellbeing systems to improve quality of life as patients start their recovery from COVID-19 that is involving immune-nutrition could be an alternative way to promote immunity and increase health statues [2]. Ongoing research is elaborating the role of the essential nutrients that may play a role in boosting up immunity and public health.
Part I: Immuno-fortified food

Garlic (strongest food as an immune booster)

Garlic is known for its ability to fight the infections caused by the viruses, bacteria and fungi [3]. Garlic might help in lowering blood pressure and reduce arteriosclerosis [4]. Its enhancement properties in the immune system appears to become from the adaptive focus of sulfur-containing compounds, such as allicin, therefore garlic thought to have more than 70 other medical benefits [5].

Onions (the second most powerful food as an immune booster)

Onions are one of the most used natural remedies to combat the respiratory infections and enhancing the immune system for its antiseptic properties and the presence of many vitamins, mineral salts, sulphur, magnesium, iron and sodium, it fights many diseases such as Diabetes Mellitus, heart disease, other infectious diseases, digestive problems and more than 40 other medicinal benefits [6]. 


\section{Citrus fruits}

These are rich in vitamin $\mathrm{C}$ which include grapefruit, oranges, tangerines, and lemons, among others. Vitamin $\mathrm{C}$ is established to help boost the immune system and it has been believed to increase the production of white blood cells which play a crucial rule in fighting variable infections [7].

Since the human body does not produce or store vitamin C, daily consumption is essential to maintain the health of the body. An example of other foods rich in vitamin $C$ includes: tomatoes, potatoes, strawberries, green, red peppers, broccoli, kiwi, and others [8].

\section{Broccoli and spinach}

Broccoli is rich in vitamins and minerals, especially vitamins A, $\mathrm{C}$ and $\mathrm{E}$, as well as many other antioxidants and fibers. It is one of the healthiest vegetables [9]. It has been recommended to cook broccoli as little as possible to enjoy its benefit. Spinach has been established to be rich with many antioxidants and beta-carotene, which may increase the ability of the immune system to fight infection [10]. As same as broccoli, spinach has been observed to be healthier when it is cooked as little as possible to preserve nutrients. However, mild cooking enhances vitamin A and allows the transport of other nutrients from oxalic acid [11].

\section{Red pepper}

It has been revealed that the red pepper contains double amount of the vitamin $\mathrm{C}$ which found in the citrus fruits. It is also a rich source of beta-carotene [12]. In addition, to its rule in the boosting the immune system, Vitamin $\mathrm{C}$ might help to keep the skin healthy. It has been established that Beta-carotene helps to keep skin clean [13].

\section{Almonds and other nuts}

Almonds contain vitamin $\mathrm{E}$ which is significant to a healthy immune system. It is a fat-soluble vitamin, which means that it needs to absorb fat properly [14]. Nuts, like almonds, they are full of vitamin $\mathrm{E}$ and also have healthy fats that it is rich with unsaturated fatty acids such as Omega-3. It has been shown that almonds and nuts might prevent and combat colds [14].

\section{Chicken soup}

Chicken soup has been shown to improve the cold symptoms and it might help to protect against the development of the respi- ratory diseases. Poultry, such as: chicken, turkey, and ducks are established to be rich in vitamin B6 which is one of B complex vitamins [15].

Vitamin B6 plays a significant role in many chemical reactions that take place in human body. It has a crucial rule for the formation of new and healthy red blood cells. Broth made from boiled chicken bones contains: gelatin, chondroitin and other nutrients which have been shown to be beneficial for the healing of the intestine [16].

\section{Green tea and black tea}

Green and black tea are containing flavonoids which are a type of antioxidant. It contains high levels of Epigallocatechin Gallate (EGCG), a powerful antioxidant. EGCG has been shown to boost the function of the immune system [17]. Green tea has been shown to be a good source of L-theanine. Green tea might help in the production of anti-microbial compounds (T cells) [18].

\section{Honey}

Honey contains antioxidants, for example: secondary plant materials, flavonoids, and ascorbic acid all are available in the natural honey [19]. It has been observed that it improves the levels of white blood cells such as: lymphocytes (both T and B cells which produce are producing plasma cells that developing antibodies), eosinophils, neutrophils, monocytes, and natural killer cells which are the essential cells of immune response to inflammation [20].

\section{Mushrooms}

Numerous investigations have been led to explain the antitumor activity of mushrooms. Therapeutic mushrooms, for example, Ganoderma lucidum and Agaricus bisporus have been proposed as a novel treatment that may improve malignant growth treatment and patients' endurance [21]. Medicinal mushrooms are accounted for to have antimicrobial, hepatoprotective, and anticancer properties. It is settled that mushrooms are proficient at invulnerable adjustment and influence hematopoietic, macrophages, $\mathrm{T}$ and $\mathrm{B}$ lymphocytes and dendritic cells [22].

\section{Yogurt}

It has been shown that the consumption of large amount of yogurt particularly among groups of patients suffering from lowered immunity, such as the elderly might activate their immune re- 
sponse [23]. Subsequently, this might increase the resistance of the immune system to many diseases such as: infection, asthma, and others. It has been established that consumption of yogurt which containing probiotics (like Lactobacillus) might raise the levels of normal Natural Killer cells, interleukins, and titters of antibodies; which might help in improvement of the immune system [24]. Prebiotics and probiotics such as fructooligosccharids (FOS) and various lactobacilli strains to improve gut dysbiosis and 'thereby improving overall immune response in such patients.

Improving gut health via gut microbiota of the people and patients is recommending with beneficial bacteria which have functional properties including particular probiotics, for example, Bifidobacterium breve M4A and different lactobacilli strains to improve gut dysbiosis and improving immune reaction in such patients. This may improve recovery patients particularly who are infected with COVID-19 [25]. Prebiotics and probiotics work by modulating immunity, including anti-inflammatory property. Moreover, beneficial bacteria can downregulate expression of ACE2 in murine gut, correlated conversely with COVID-19 quantity in fecal probs from patients [25].

\section{Human milk oligosaccharides}

Human milk oligosaccharides (HMO) can imitate the composition of the viruses' specific receptor on human cells, binding to the virus and stop it from attacking with its objective cells [26]. The possibility interaction between histo-blood group antigens (HBGs) and noroviruses, which is detected as norovirus receptors, could be affected by HMO because of likenesses to and HBGs structure and excess in human breastmilk [27]. HMO particularly lacto-Nfrucopentaose can binding to the norovirus that it hinders to tie to human mucins and guard against norovirus diseases. Symptoms of norovirus infectious are recognized by vomiting and diarrhea. In any case, infection symptoms can be more severe in children, the immunocompromised and the elderly people [28].

Fish

Selenium which has been found in fish, has been concluded to help the white blood cells in production of cytokines, which in turn expel the respiratory viruses from the body [29]. Fish is rich in omega-3 oil which helps in reduction of inflammation, increase air flow to the lungs and might protect them from colds and infections. In addition, these fatty acids are effective in: improving mood, in- creasing the body activity, strengthening memory and protecting the body from cardiovascular disease [30].

\section{Red meat}

Zinc deficiency is clearly common among adults in America, especially vegetarians due to lack of consumption of beef which is considered a major source of this mineral that strengthening immunity [31].

Avocado

Avocado contains: monounsaturated fatty acids, iron, and zinc along with several different vitamins such as $\mathrm{E}$ and $\mathrm{C}$ that interfere with stimulating the body to produce antibodies in abundance amounts which fights and cure the microbial invasions to the body [32].

\section{Part II: Vitamins and minerals that boost immune response} Vitamin D

Vitamin $\mathrm{D}_{3}$, one of the important vitamins which plays a large role in the function of the immune system. It has been observed in several scientific studies that people who have a deficiency in the level of this vitamin are more likely to develop the following diseases: respiratory infections (viral and bacterial), chronic bronchial diseases, and bronchial asthma [33].

Subsequent to contemplating global information from the novel COVID-19 pandemic, scientists have found a solid connection between serious vitamin D deficiency and death rates [34]. A correlation had been clarified between vitamin $\mathrm{D}$ levels and cytokine storm which a hyperinflammatory condition brought about by an overactive immune response, when cytokine storm can seriously harm and damage lungs tissue and lead to intense respiratory trouble disorder and death in patients. In this manner, that having recommended levels of vitamin $\mathrm{D}$ could save patients against serious health problem, including death from COVID-19 [35].

Therefore, some research has stated that maintaining the level of vitamin D within the normal level or using supplements containing vitamin $\mathrm{D}$ within the normal can help reduce the incidence of acute respiratory diseases [36].

Vitamin D is available in these foods: fish (salmon, sardines, tuna), eggs, mushrooms, fortified food) [37]. A very important note to mention that vitamin D may prevent infection from coronavirus (COVID-19) [34]. 
In addition, there has been recent evidences on the relationship between vitamin D supplementation and the low chance of COVID19 infection [38]. Despite of that, it has been found in several scientific studies that a lack of vitamin D increases the risk of developing respiratory diseases [39].

\section{Vitamin A}

One of the important vitamins which is necessary in the human body. It plays a crucial role in cell division and reproduction, fetal development, vision, as well as immune system in the human body [40]. It has been shown that it plays a significant role in the development of the lungs and alveoli [41]. It has been proved that it is an essential element to support the immune system because of its crucial role as an anti-inflammatory [42]. Researchers have proven the significance of vitamin A in increasing the defensive ability of the immune system in many infections. Vitamin A is abundant in these products: red pepper, carrots, sweet potatoes, dark-leaf vegetables, pumpkins, lettuce and dry herbs [43].

Zinc

Zinc has been shown to play a central role in the functioning of the immune system, and its deficiency exposes humans to many diseases [44]. In addition, it has been noted that acute and chronic deficiency of zinc associated with increasing the susceptibility to infections and its onset. Foods containing zinc include the following: red meat, eggs, nuts, dairy products and dark chocolate [45].

\section{Vitamin C}

One of the important vitamins in the human body and is considered an antioxidant. It plays an important role in the function of the immune system [46]. Scientific studies have proven that vitamin $\mathrm{C}$ deficiency weakens immunity and increases susceptibility to inflammation. The use of vitamin $C$ has been noted to be associated with increase the ability to treat and prevent respiratory infections [46].

\section{Vitamin E}

Vitamin $\mathrm{E}$ is an antioxidant and plays a pivotal order in building up a good immunity [47]. It has been stated that to boost the immunity of human body, the following group of foods are highly helpful: almonds, spinach, broccoli, parsley, avocado, and soybeans [48].

These types of food are rich in vitamin E. The Vitamin E antioxidant properties via destruction of free radicals which are harmful to boost the immune system [49].
Part III: Immuno-elevated herbs

\section{Turmeric}

This bright yellow spice has been used for many years as an anti-inflammatory in the treatment of both arthritis and rheumatoid arthritis. It has been shown to be useful in treatment of influenza and respiratory infections [50]. It has been observed that high concentrations of curcumin, (which provides the turmeric its distinctive color) might help in reduction of exercise damage [51].

\section{Ginger}

Ginger (Zingiber officinale) has been observed to reduce the inflammation in general as well as sore throats. It might help in the relieve of both nausea and chronic pain. Ginger has been noted to has cholesterol-lowering properties [52].

\section{Ginseng}

Ginseng or Korean ginseng (Panax ginseng meyer) is one of the most well-known medicinal herbs and has been utilized as cancer remedial agent plant [53]. The anti-cancer activity of ginseng saponins are ascribed essentially to the existence of ginsenosides [54]. Ginsenosides are one of the pharmaceutical substances which apply their anticancer impacts by tweaking of different flagging pathways, including regulation of cell multiplication go between growth factors and vascular endothelial development factor, regulation of p53 pathway and tumor suppressors of cell cycle regulation and apoptosis. Hence, ginseng has capability in the treatment of different human diseases [55]. A clinical report showed the helpful impacts of ginseng administration of vaccinated mice with red ginseng gave improved cross-protection against antigenically distinct $\mathrm{H} 1 \mathrm{~N} 1$ and $\mathrm{H} 3 \mathrm{~N} 2$ flu infections and ginseng extracts were found antiviral to affect flu infection [56].

\section{Liquorice}

Liquorice is the English name of Glycyrrhiza glabra that it might be an effective medicinal substance for COVID-19 [57]. Glycyrrhizin has different biological activities and pharmacological impacts [58]. A pharmacological activity of the glycyrrhizin including binding angiotensin-converting enzyme (ACE2), downregulating proinflammatory cytokines, repressing the accumulation of intracellular reactive oxygen species [58].

\section{Conclusion}

COVID-19 pandemic is an important health dilemma that has been evolved since late December 2019 right now. Up to date, there 
is no valid authenticated vaccine or drug for COVID-19.

One of the important rules for fighting COVID19 by trying to keep the level of immune system is high. Thus, general population need to improve their immune system with nutrients and food supplements.

In addition, it is crucial to keep adhering to the instructions of the Health Authorities in each country which is generally emphasizes on: commitment to social distancing, using personal protective equipment (PPE), avoiding crowded places, using of adequate hygiene for hand, avoid contact or be away from people with symptoms and signs of respiratory diseases, keep adequate and fundamental nutrient, and ask for health assistant when it is required.

\section{Conflicts of Interest}

The authors declare no conflicts of interest.

\section{Funding Support}

This is a self-funded work.

\section{Bibliography}

1. Butler MJ and Barrientos RM. "The impact of nutrition on COVID-19 susceptibility and long-term consequences". Brain, Behavior, and Immunity 87 (2020): 53-54.

2. Kalantar-Zadeh K and Moore LW. "Impact of nutrition and diet on COVID-19 infection and implications for kidney health and kidney disease management". Journal of Renal Nutrition 30.30 (2020): 179-181.

3. Wolde T., et al. "Anti-bacterial activity of garlic extract against human pathogenic bacteria". Journal of Pharmacovigilance 6.253 (2018): 2-8.

4. Wlosinska M., et al. "The effect of aged garlic extract on the atherosclerotic process-a randomized double-blind placebocontrolled trial". BMC Complementary Medicine and Therapies 20 (2020): 1-10.

5. Percival SS. "Aged garlic extract modifies human immunity". The Journal of Nutrition 146.2 (2016): 433S-436S.

6. Lee J-B., et al. "Anti-influenza A virus effects of fructan from Welsh onion (Allium fistulosum L.)". Food Chemistry 134.4 (2012): 2164-2168.
7. Baba E., et al. "Evaluation of Citrus limon peels essential oil on growth performance, immune response of Mozambique tilapia Oreochromis mossambicus challenged with Edwardsiella tarda". Aquaculture 465 (2016): 13-18.

8. Llorens E., et al. "Enhancement of the citrus immune system provides effective resistance against Alternaria brown spot disease". Journal of Plant Physiology 170.2 (2013): 146-154.

9. Kaczmarek JL., et al. "Broccoli consumption affects the human gastrointestinal microbiota". The Journal of Nutritional Biochemistry 63 (2019): 27-34.

10. Song T., et al. "Functional analysis of spinach CONSTITUTIVE EXPRESSOR OF PATHOGENESIS-RELATED GENES5 (SoCPR5) in plant immunity". European Journal of Plant Pathology 150.2 (2018): 515-520.

11. Deng Z., et al. "Broccoli-derived nanoparticle inhibits mouse colitis by activating dendritic cell AMP-activated protein kinase". Molecular Therapy 25.7 (2017): 1641-1654.

12. Reda F., et al. "Use of red pepper oil in quail diets and its effect on performance, carcass measurements, intestinal microbiota, antioxidant indices, immunity and blood constituents". Animal 14.5 (2020): 1025-1033.

13. Bortolotti M and Porta S. "Effect of red pepper on symptoms of irritable bowel syndrome: preliminary study". Digestive Diseases and Sciences 56.11 (2011): 3288-3295.

14. Burns AM., et al. "Diet quality improves for parents and children when almonds are incorporated into their daily diet: a randomized, crossover study". Nutrition Research 36.1 (2016): 80-89.

15. Marsh SB. "Soup is a healthy choice; chicken noodle soup really does help cure a cold". Letters to the Editor [Dorothy Paulsen] Postville Project Documents (2019): 226.

16. Lavine JB. "Chicken soup or Jewish medicine". Chest 119.4 (2001): 1295-1295.

17. Qian Y., et al. “ (-)-Epigallocatechin gallate-loaded polycaprolactone scaffolds fabricated using a 3D integrated moulding method alleviate immune stress and induce neurogenesis". Cell Proliferation 53.1 (2020): e12730. 
18. Ravikumar C. "Review on herbal teas". Journal of Pharmaceutical Sciences and Research 6.5 (2014): 236.

19. Oduwole O., et al. "Honey for acute cough in children. Cochrane Database of Systematic Reviews 4 (2018): CD007094.

20. Kwong WK., et al. "Immune system stimulation by the native gut microbiota of honey bees". Royal Society Open Science 4.2 (2017): 170003.

21. Wasser SP. "Medicinal mushrooms in human clinical studies. Part I. Anticancer, oncoimmunological, and immunomodulatory activities: a review". International Journal of Medicinal Mushrooms 19.4 (2017): 279-317.

22. Guggenheim AG., et al. "Immune modulation from five major mushrooms: application to integrative oncology". Integrative Medicine: A Clinician's Journal 13.1 (2014): 32.

23. El-Abbadi NH., et al. "Yogurt: role in healthy and active aging". The American Journal of Clinical Nutrition 99.5 (2014): 1263S$1270 \mathrm{~S}$.

24. Lollo PCB., et al. "Probiotic yogurt offers higher immune-protection than probiotic whey beverage". Food Research International 54.1 (2013): 118-124.

25. Dhar D and Mohanty A. "Gut microbiota and Covid-19-possible link and implications”. Virus Research 285 (2020): 198018.

26. Etzold S and Bode L. "Glycan-dependent viral infection in infants and the role of human milk oligosaccharides". Current Opinion in Virology 7 (2014): 101-107.

27. Koromyslova A., et al. "Human norovirus inhibition by a human milk oligosaccharide”. Virology 508 (2017): 81-89.

28. Derya SM., et al. "Biotechnologically produced fucosylated oligosaccharides inhibit the binding of human noroviruses to their natural receptors". Journal of Biotechnology 318 (2020): 31-38.

29. Avery JC and Hoffmann PR. "Selenium, selenoproteins, and immunity”. Nutrients 10.9 (2018): 1203.

30. Ruxton C. "The benefits of fish consumption". Nutrition Bulletin 36.1 (2011): 6-19.
31. Sandstead HH and Freeland-Graves JH. "Dietary phytate, zinc and hidden zinc deficiency". Journal of Trace Elements in Medicine and Biology 28.4 (2014): 414-417.

32. Piccirilli A., et al. "Medicament comprising a peptide extract of avocado, which is intended for the treatment and prevention of illnesses that are linked to an immune system deficiency or oxidative stress or skin ageing or dry skin". Patent No: US 9,089,576 B2 (2015).

33. Ali NS and Nanji K. "A Review on the Role of Vitamin D in Asthma”. Cureus 9.5 (2017): e1288-e1288.

34. Ilie PC., et al. "The role of vitamin D in the prevention of coronavirus disease 2019 infection and mortality". Aging Clinical and Experimental Research 32 (2020): 1195-1198.

35. Daneshkhah A., et al. "The possible role of Vitamin D in suppressing cytokine storm and associated mortality in COVID-19 patients". MedRxiv (2020).

36. Martineau AR., et al. "Vitamin D supplementation to prevent acute respiratory infections: individual participant data metaanalysis". Health Technology Assessment 23.2 (2019): 1-44.

37. Christakos S., et al. "Vitamin D: metabolism". Rheumatic Disease Clinics 38.1 (2012): 1-11.

38. Grant WB., et al. "Evidence that vitamin D supplementation could reduce risk of influenza and COVID-19 infections and deaths". Nutrients 12.4 (2020): 988.

39. Rondanelli M., et al. "Self-care for common colds: the pivotal role of vitamin $\mathrm{D}$, vitamin $\mathrm{C}$, zinc, and echinacea in three main immune interactive clusters (physical barriers, innate and adaptive immunity) involved during an episode of common colds-practical advice on dosages and on the time to take these nutrients/botanicals in order to prevent or treat common colds". Evidence-Based Complementary Alternative Medicine Review 2018 (2018): 1-36.

40. Wirth JP., et al. "Vitamin A supplementation programs and country-level evidence of vitamin A deficiency". Nutrients 9.3 (2017): 190.

41. Timoneda J., et al. "Vitamin A Deficiency and the Lung". Nutrients 10.9 (2018): 1132. 
42. Erkelens MN., et al. "Intestinal macrophages balance inflammatory expression profiles via vitamin A and Dectin-1-mediated signaling". Frontiers in Immunology 11 (2020): 551.

43. Huang Z., et al. "Role of vitamin A in the immune system". Journal of Clinical Medicine 7.258 (2018): 1-16.

44. Haase H and Rink L. "Functional significance of zinc-related signaling pathways in immune cells". Annual Review of Nutrition 29 (2009): 133-152.

45. Bonaventura P., et al. "Zinc and its role in immunity and inflammation”. Autoimmunity Reviews 14.4 (2015): 277-285.

46. Carr AC and Maggini S. "Vitamin C and immune function". Nutrients 9.11 (2017): 1211.

47. Blount BC., et al. "Vitamin E acetate in bronchoalveolar-lavage fluid associated with EVALI". New England Journal of Medicine 382.8 (2020): 697-705.

48. Reboul E. "Vitamin E bioavailability: Mechanisms of intestinal absorption in the spotlight". Antioxidants 6.4 (2017): 95.

49. Poprac P., et al. "Targeting free radicals in oxidative stress-related human diseases". Trends in Pharmacological Sciences 38.7 (2017): 592-607.

50. Dalal R., et al. "Effect of turmeric powder on egg quality, gut morphology, ecology and on immune system of laying hen: A review". Journal of Entomology and Zoology Studies 6.3 (2018): 978-982.

51. Naderi M., et al. "Effects of dietary inclusion of turmeric (Curcuma longa) and cinnamon (Cinnamomum verum) powders on performance, organs relative weight and some immune system parameters in broiler chickens". Poultry Science Journal 2.2 (2014): 153-163.

52. Sukumaran V., et al. "Role of dietary ginger Zingiber officinale in improving growth performances and immune functions of Labeo rohita fingerlings". Fish and Shellfish Immunology 57 (2016): 362-370.

53. Chong-Zhi W., et al. "Red ginseng and cancer treatment". Chinese Journal of Natural Medicines 14.1 (2016): 7-16.
54. Yu JS., et al. "Bioactivity-guided isolation of ginsenosides from Korean Red Ginseng with cytotoxic activity against human lung adenocarcinoma cells". Journal of Ginseng Research 42.4 (2018): 562-570.

55. Gao J-L., et al. "Ginseng saponin metabolite 20 (S)-protopanaxadiol inhibits tumor growth by targeting multiple cancer signaling pathways". Oncology Reports 30.1 (2013): 292-298.

56. Yoo D-G., et al. "Protective effect of Korean red ginseng extract on the infections by H1N1 and H3N2 influenza viruses in mice". Journal of Medicinal Food 15.10 (2012): 855-862.

57. Xu J and Zhang Y. "Traditional Chinese medicine treatment of COVID-19". Complementary Therapies in Clinical Practice 39 (2020): 101165.

58. Pan L., et al. "Pharmacologic perspective: glycyrrhizin may be an efficacious therapeutic agent for COVID-19". International Journal of Antimicrobial Agents 55.6 (2020): 105995.

\section{Volume 4 Issue 8 August 2021 \\ (C) All rights are reserved by Majid Mohammed Mahmood and Zenab Ghanim Younus Al-Ameen.}

